\title{
The tie effect in simple arithmetic: An access-based account
}

\author{
JO-ANNE LEFEVRE, TINA SHANAHAN, and DIANA DESTEFANO \\ Carleton University, Ottowa, Ontario, Canada
}

\begin{abstract}
Simple arithmetic problems with repeated operands (i.e., ties such as $4+4,6 \times 6,10-5$, or $49 \div 7$ ) are solved more quickly and accurately than similar nontie problems (e.g., $4+5,6 \times 7,10-6$, or 48 $\div 6$ ). Further, as compared with nonties, ties show small or nonexistent problem-size effects (whereby problems with smaller operands such as $2+3$ are solved more quickly and accurately than problems with larger operands such as $8+9$ ). Blankenberger (2001) proposed that the tie advantage occurred because repetition of the same physical stimulus resulted in faster encoding of tie than of nontie problems. Alternatively, ties may be easier to solve than nonties because of differences in accessibility in memory or differences in the solution processes. Adults solved addition and multiplication (Experiment 1) or subtraction and division (Experiment 2) problems in four two pure formats (e.g., $4+4$, FOUR + FOUR) and two mixed formats (e.g., $4+$ FOUR, and FOUR +4 ). Tie advantages were reduced in mixed formats, as compared with pure formats, but the tie $\times$ problem-size interaction persisted across formats. These findings support the view that tie effects are strongly related to memory access and are influenced only moderately by encoding factors.
\end{abstract}

Research concerning how people solve simple arithmetic problems such as $3 \times 4$ or $16-8$ is central to understanding mathematical cognition. Certain effects are well established. For example, children and adults solve problems with smaller operands (e.g., $2+3,4 \times 2,7-3$, $15 \div 3$ ) more quickly and accurately than problems with larger operands (e.g., $9+8,6 \times 7,15-9,72 \div 8$; Ashcraft, 1995; Groen \& Parkman, 1972; LeFevre, Bisanz, Daley, Buffone, Greenham, \& Sadesky, 1996; LeFevre, Sadesky, \& Bisanz, 1996; Miller, Perlmutter, \& Keating, 1984; Penner-Wilger, Leth-Steensen, \& LeFevre, 2002). Similarly, researchers have reported an advantage for tie problems. In addition and multiplication, ties are problems with repeated operands (e.g., $3+3,9 \times 9$ ). Subtraction and division ties are defined as the complements of tie addition and multiplication problems (e.g., $6-3,81 \div 9$ ). Tie problems such as $9+9$ and $16-8$ are solved more quickly and accurately than comparable nonties such as $9+8$ or 16 - 7 (Campbell \& Gunter, 2002; Groen \& Parkman, 1972; LeFevre \& Liu, 1997; Miller et al., 1984). Furthermore, problem size and tie effects interact, such that the problem-size effect is smaller for ties than for nonties (Campbell \& Gunter, 2002; Groen \& Parkman, 1972; LeFevre \& Liu, 1997; Miller et al., 1984). Despite approximately 30 years of research, however, considerable

This research was supported by a grant from the Natural Sciences and Engineering Research Council of Canada to J. L. Portions of the data were presented at the annual meetings of the Canadian Society for Brain, Behaviour, and Cognitive Science in Vancouver, May-June 2002. Correspondence concerning this article should be addressed to J.-A. LeFevre, Centre for Applied Cognitive Research, Department of Psychology, Carleton University, Ottawa, ON, K1S 5B6 Canada (email: jo-anne_lefevre@carleton.ca). controversy remains about the source of problem-size and tie effects.

Theoretical accounts of both problem-size and tie effects attribute differences in solution latencies and accuracy across problem categories to variability in accessibility, with ties and smaller problems assumed to be more accessible in the mental representation than nonties and larger problems (Ashcraft, 1992, 1995; Campbell, 1995; Campbell \& Gunter, 2002). These access-based accounts can be contrasted with an encoding-based explanation for the tie advantage in addition and multiplication offered by Blankenberger (2001). He proposed that the tie advantage occurs because repetition of the same physical stimulus results in faster encoding of tie than of nontie problems. To test this proposal, Blankenberger compared latencies of addition and multiplication ties and nonties across four formats, the traditional digit-digit format (e.g., $3+3$ ), a word-word format (e.g., THREE + THREE), and two mixed formats (e.g., $3+$ THREE and THREE +3 ). Blankenberger found that the tie advantage (i.e., faster latencies on ties than on nonties) disappeared completely in the two mixed formats. These data appear to provide strong and convincing evidence that the tie effect is due entirely to an encoding advantage.

Blankenberger's (2001) characterization of the tie effect was incomplete, however, in that he did not consider that it comprises both a tie advantage and a tie $\times$ problemsize interaction. Typically, ties show no problem-size effect at all (Campbell \& Gunter, 2002; Groen \& Parkman, 1972) or a much smaller problem-size effect than nonties (LeFevre, Bisanz, et al., 1996; LeFevre \& Liu, 1997; LeFevre, Sadesky, \& Bisanz, 1996; LeFevre, Smith-Chant, Hiscock, Daley, \& Morris, 2003; Miller et al., 1984). Furthermore, the solution latencies of small ties and small 
nonties do not differ, suggesting that an encoding advantage is not a primary source of the tie effect (Campbell \& Gunter, 2002). In the present research, we have examined both tie advantages and tie $\times$ size interactions across all four arithmetic operations: addition, subtraction, multiplication, and division. In subtraction and division, tie problems were defined as the complements of the tie problems in addition and multiplication (e.g., 8 - 4; $4 \div 2$; Campbell, 1997; Campbell \& Xue, 2001).

Explanations for effects of ties and problem size that are based on differential accessibility across problems fall into three categories: (1) familiarity, (2) interference, and (3) variability in solution approaches. Note that these explanations are not mutually exclusive. According to familiarity explanations, accessibility varies with practice such that problem size and tie effects are related to the frequency with which problems are encountered. Thus, large problems are solved more slowly than small problems because they receive less practice, and therefore the connections between operands and answers in memory are weaker (Ashcraft \& Christy, 1995; Geary, 1996; Zbrodoff, 1995). Similarly, participants may have solved ties more frequently than nonties. In support of familiarity explanations, Siegler and Shrager (1984) found that parents presented tie addition problems to preschoolers more frequently than they presented most other types of problems. Tabulation of the frequency of ties versus nonties in textbooks, however, did not support the view that children experience ties more frequently than nonties (Ashcraft \& Christy, 1995; Hamann \& Ashcraft, 1986), although small problems were presented much more frequently than large problems (cf. Geary, 1996). To account for the tie $\times$ size interaction with a familiarity explanation, one would have to assume that large ties are presented more frequently than large nonties, but that small ties and small nonties are presented equally often.

According to interference explanations of problemsize effects, slower latencies on large problems reflect the internal mental representation of these problems such that larger problems interfere more with one another than do smaller problems (Campbell, 1995). In a simulation model of addition and multiplication, Campbell (1995) defined ties and nonties as separate categories of problems and then allowed interference to be greater within categories than between categories. Thus, because the tie category included relatively few problems, tie problems received less inhibitory input than nontie problems. Using this mechanism, Campbell's simulation produced a tie advantage in multiplication, although not in addition. It was not clear whether the simulation would produce an interaction between tie status and problem size, however, because the interaction was not tested.

The third proposed source of the problem-size effect is related to variability in the selection of solution procedures across problems. Researchers have found that adults solve arithmetic problems via both direct retrieval from memory and various nonretrieval alternatives. For example, people reported solving problems such as $4+3$ by counting $(5,6,7)$, or problems such as $9 \times 6$ by accessing related facts (e.g., $10 \times 6-6$; Campbell \& Xue, 2001; Hecht, 1999; LeFevre, Bisanz, et al., 1996; LeFevre \& Liu, 1997; Smith-Chant \& LeFevre, 2003). Because participants report using solution procedures other than direct retrieval from memory more frequently on large than on small problems, and because such procedures are often slower than direct retrieval, selection of procedures has been proposed as one source of the problem-size effect (Campbell \& Xue, 2001; Groen \& Parkman, 1972; LeFevre, Sadesky, \& Bisanz, 1996; Smith-Chant \& LeFevre, 2003).

Campbell and Gunter (2002; Experiment 1) reanalyzed data that were collected by Campbell and Xue (2001) for Asian- and Canadian-educated students solving addition, multiplication, subtraction, and division problems in digit-digit format. They found support for an accessibility explanation of the tie $\times$ size interaction. First, the pattern of the tie $\times$ size interaction corresponded to earlier accounts in that (1) there was no difference in solution latencies for ties versus nonties on small problems, (2) for students educated in China, there was no tie advantage at all in addition, a result that is inconsistent with an encodingbased explanation, and (3) participants showed tie advantages and tie $\times$ size interactions in subtraction (e.g., $8-4$ vs. $7-4$ ) and division (e.g., $16 \div 4$ vs. $12 \div 4$ ) as well as in addition and multiplication. Tie advantages in subtraction and division cannot be accounted for with an explanation that is based on physical encoding because ties in these operations do not involve repetition of the same stimulus. Furthermore, participants' self-reports of their solution procedures indicated that a substantial portion of the tie $\times$ size interaction was due to a greater use of nonretrieval solutions on large nonties, as compared with large ties, across all four operations. On the basis of these findings, Campbell and Gunter argued against an encoding explanation and in favor of an accessibility account of the tie advantage and the tie $\times$ size interaction, that is, more efficient access to memory for tie versus nontie problems.

LeFevre et al. (2003) also found support for the hypothesis that the tie $\times$ size interaction was related to differential selection of procedures. Participants $(n=48)$ solved single-digit multiplication problems and provided a description of their solution procedure after each problem. Three groups of participants were identified. $R e$ trievers $(n=16)$ reported using memory retrieval on $100 \%$ of trials. Occasional procedure users $(n=16)$ reported using procedures other than retrieval, such as accessing related facts, on an average of $5 \%$ of trials. Frequent procedure users $(n=16)$ reported using procedures other than retrieval on an average of $25 \%$ of trials. Both the occasional and frequent procedure users showed the typical interaction between tie status and problem size for multiplication. Latencies on both tie and nontie problems increased with problem size, but the increase was much greater on nonties than on ties. In contrast, retrievers showed only a main effect of tie status, suggesting that an explanation based on encoding facilitation was plausible 
for this group. Thus, for procedure users, interactions between tie status and problem size may indicate that they used procedures other than retrieval more frequently on nontie than on tie problems.

In summary, Blankenberger's (2001) encoding explanation for the tie effect does not address the source of the tie $\times$ size interaction. Whenever problem size has been considered in comparisons between tie and nontie problems, an interaction has been observed between tie status and problem size. The reduced problem-size effect for ties as compared with nonties is unlikely to be explained by encoding facilitation, because small ties and nonties are often solved equally quickly. Thus, further consideration of the tie $X$ size interaction as a function of format is necessary to understand the source of the tie effect.

Campbell and Gunter (2002; Experiment 2) replicated Blankenberger's (2001) finding that the tie advantage was eliminated in mixed format conditions for multiplication problems, but they proposed an alternative explanation. They argued that the design used by Blankenberger confounded practice with tie status. Blankenberger presented nontie problems twice (e.g., $3 \times 4$ and $4 \times 3$ ), whereas ties were tested once. Hence, nontie problems received more practice than tie problems, on the assumption that presentation of both orders of a nontie results in almost as much facilitation as presenting the same problem twice (Rickard \& Bourne, 1996; Rickard, Healy, \& Bourne, 1994). Campbell and Gunter found support for a practice account of reduced tie effects: When their participants had practiced both orders of nontie problems, the tie advantage in latencies was reduced as compared with the first presentation of the nontie problems. However, this practice effect occurred in all four formats, so it cannot alone account for the complete reduction in the tie advantage in mixed formats. Campbell and Gunter also showed that, for multiplication problems, performance on small ties in the mixed formats (e.g., FOUR $\times 4$ ) was actually worse than for comparable nonties (e.g., FOUR $\times 5$ ). This dramatic reversal in the tie advantage, combined with the practice effect, was shown to produce the same overall pattern as found by Blankenberger, that is, an absence of a tie advantage in the mixed format conditions.

Campbell and Gunter (2002) suggested that the reversed tie effect for small multiplication problems occurred because the mixed format condition produced interference. They cited Butterworth, Zorzi, Girelli, and Jonckheere (2001), who claimed that, for addition, participants performed a number comparison before solving the problems. In the COMP model presented by Butterworth et al. (2001), this magnitude comparison occurs only after a number identification stage, during which abstract numerical identities are established and the cardinal magnitude of the operands is automatically activated. The model assumes that magnitude comparison is circumvented in the case of ties in which a physical match judgment is made. According to this view, the surface-form mismatch (i.e., FOUR vs. 4) would interfere at the identification stage and cause the numerical comparison to be performed for ties as well as for nonties. In the present research, participants solved problems in all four operations in all four formats. If a mismatch of surface forms for ties produces interference, as proposed by Campbell and Gunter, we expected to find reversed tie advantages for both addition and multiplication problems.

Blankenberger (2001) tested only addition and multiplication problems, in which repetition of the physical stimulus is a possible explanation for tie effects. Tie effects are also found in subtraction (Campbell \& Gunter, 2002) and division (Campbell, 1997, 1999; Campbell \& Gunter, 2002), but such effects cannot be due to encoding. A tie advantage in subtraction and division could be due to a form of facilitation in which the match between the operand and the answer results in facilitation of answer production (e.g., $16 \div$ FOUR $=$ FOUR). However, an account of the tie effect for subtraction and division that is based on answer facilitation does not explain a tie $\times$ size interaction for these operations. Thus, to the extent that the tie effect consists of both a tie advantage and a tie $\times$ size interaction, the elimination of the overall tie advantage found by Blankenberger may address only one aspect of why ties are easier to solve than nonties. The present research was designed to address this limitation by analyzing patterns of latencies with problem size included as a variable, and by extending the research to subtraction and division problems.

\section{EXPERIMENT 1}

In Experiment 1, we have used the same methodology as Blankenberger (2001) and collected latency and accuracy data across the four formats for addition and multiplication. We hypothesized that, in the digit-digit condition, there would be both a tie advantage and a tie $\times$ size interaction. Specifically, based on all the existing patterns shown for ties versus nonties in digit formats, large nonties should always be solved more slowly than large ties, whereas small ties and small nonties should have similar latencies. A second hypothesis was that, to the extent that the tie advantage is due to encoding facilitation, the tie advantage should be larger in the word-word condition than in the digit-digit condition because the overall slower latencies with word-word format provide more opportunity for encoding facilitation to influence latencies. This prediction is based on the assumption that word format influences encoding and response production (Noël, Fias, \& Brysbaert, 1997; Noël, Robert, \& Brysbaert 1998; cf. Campbell, 1998). Third, we predicted that the tie $\times$ size interaction would be preserved across the format conditions on the assumption that this interaction reflects variability in the accessibility of answers, either through differential use of solution procedures or through differential efficiency of retrieval processes for ties versus nonties. Fourth, based on Campbell and Gunter's (2002) view that a number comparison process is disrupted in the mixedformat conditions, we predicted a reversed tie advantage on small problems for addition and multiplication. 


\section{Method}

\section{Participants}

Eight graduate and advanced undergraduate students ( 6 females and 2 males) participated in this experiment. They ranged in age from 20 to 35 years, with a median age of 23.5. They were paid $\$ 90$ for their participation. The participants were recruited through personal contacts. Six had been educated in Canada, one in Sweden and Canada, and one in Australia.

\begin{abstract}
Materials
Naming. To test production (naming) latencies, the 36 possible correct answers to the addition and multiplication problems were presented in random order in digit format. The participants named each digit three times, for a total of 108 naming trials per session.

Arithmetic. The participants solved all 64 addition and 64 multiplication problems with operands 2 through 9 (i.e., $2+2$ to $9+9$ and $2 \times 2$ to $9 \times 9$ ) in four different formats in each of five sessions. Operands were printed either as Arabic digits or as words in English so that all problems were presented in two pure (e.g., $4 \times 8$, FOUR $\times$ EIGHT) and two mixed (e.g., $4 \times$ EIGHT, FOUR $\times 8$ ) formats. The participants, therefore, solved 512 simple arithmetic problems (128 $\times 4$ formats), divided into 8 blocks of 64 , in each session. Format (digit-digit, word-word, digit-word, word-digit) and operation (addition, multiplication) varied randomly from trial to trial. As in Blankenberger (2001), nontie problems were presented in both orders, whereas tie problems were presented once.

Fluency test. The participants completed the addition and subtraction-multiplication subtests of the French Kit (French, Ekstrom, \& Price, 1963) as a measure of arithmetic fluency. Each subtest of this paper-and-pencil task consists of two pages of problems. The participants were instructed to solve the problems as quickly and accurately as possible and were given 2 min per page. Arithmetic fluency was measured as the total number of correct solutions on both tests combined.
\end{abstract}

Background questionnaire. The participants also completed a detailed questionnaire regarding educational background, past experience with arithmetic, self-rated arithmetic skill, and use of arithmetic strategies.

\begin{abstract}
Apparatus
The simple addition and multiplication problems were presented horizontally on a monochrome monitor connected to an IBMcompatible 80286 computer. For all problems, the sign $(+$ or $\times$ ) was centered on the screen. Stimuli were presented in a light color on a dark background. The participants responded using a headset microphone attached to a Soundblaster board. Latencies were recorded with a specialized input/output board that was accurate to the nearest millisecond. Responses were recorded by the experimenter.
\end{abstract}

\section{Procedure}

The participants completed an initial 1.5-h session and four subsequent 1-h sessions. In all sessions, they completed first the experimental task (solving 512 arithmetic problems), then the naming task. In the experimental task, all the participants solved all arithmetic problems four times each, once in each format. For the naming task, they were presented with three blocks of 36 numbers and were asked to name them. In both tasks, blocks were separated by breaks of at least $30 \mathrm{sec}$. Prior to each task, the participants were instructed to respond as quickly and accurately as possible. In the first session, following the computer tasks, the participants completed the arithmetic fluency test and the background questionnaire.

On each trial of the computer tasks, a diamond appeared for $500 \mathrm{msec}$ in the center of the screen, flashing on and off twice at 250msec intervals. On what would have been the third flash of the diamond, the arithmetic problem (e.g., $4+$ FOUR) or a number in the case of naming (e.g., 36) appeared. For the arithmetic problems, the oper- ation sign appeared at the same location as the flashing diamond. The stimulus remained on the screen until the participant responded verbally or until $10 \mathrm{sec}$ had elapsed. The experimenter recorded the participant's response. No feedback about speed or accuracy was given on any trials.

\section{Results}

\section{Questionnaire and Arithmetic Fluency}

The participants described a variety of procedures for solving simple arithmetic problems, including retrieval from memory, decomposition (e.g., $7 \times 9=[7 \times 10]-7$ ), counting backward, and a nines rule (for descriptions of the nines rules, see LeFevre, Sadesky, \& Bisanz [1996] for addition and LeFevre, Bisanz, et al. [1996] for multiplication). Most of the participants reported that they used procedures other than retrieval sometimes or often for multiplication ( 6 out of 8 ) and addition ( 8 out of 8 ) and often or always for subtraction (6 out of 8 ) and division (5 out of 8 ).

The total scores on the arithmetic fluency test for these participants (i.e., total number correct across all four pages of the test) ranged from 45 to 116 , with a mean of $79(S D=21.5)$. The typical mean for samples of undergraduates on this measure is approximately $80(S D=20$; LeFevre et al., 2003). Thus, as a group, these participants had average arithmetic skills.

\section{Naming}

The purpose of the naming task was to estimate the contribution of response production times to tie and problem-size effects in solving simple addition and multiplication problems. The participants completed a total of 4,320 naming trials. For addition, all of the even numbers from 6 to 16 occur as both tie and nontie sums, whereas the odd numbers in the answer set occur only as nontie sums, and 4 and 18 occur only as tie sums. For multiplication, only two of the tie products (16 and 36) also occur as nontie products.

Large problems were defined as those for which the product of the operands is greater than 25 . In multiplication, therefore, answers for small problems are those less than or equal to 25 and for large problems those greater than 25 . For addition, the corresponding small problem answers are the numbers 4 to 10, and the large problem answers are the numbers 12 to 18 . Eleven occurs as the answer to four small $(2+9,9+2,3+8,8+3)$ and four large $(4+7$, $7+4,5+6,6+5)$ problems.

Median correct naming latencies were calculated for each participant, separately for addition and multiplication answers. For addition, the average of these median naming times for answers to large problems was $545 \mathrm{msec}$ and for small problems it was $537 \mathrm{msec}$. For ties and nonties, average times were 545 and $540 \mathrm{msec}$, respectively. Large and small multiplication problem answers were named in 566 and $543 \mathrm{msec}$, respectively. Multiplication ties and nonties were named in 557 and $555 \mathrm{msec}$, respectively. These differences were very small relative to problem size and tie effects typically observed for simple 
arithmetic problems. Clearly, answer production did not contribute in any important way to problem size or tie effects in the arithmetic trials. Therefore, it was not considered necessary to correct for naming latencies when analyzing arithmetic solution times.

\section{Arithmetic}

The participants solved a total of 20,480 addition and multiplication problems. The first session was treated as practice and was excluded from the analysis. For the remaining four sessions, 457 trials were errors and 544 were invalid, due to either inadvertent voice key triggers or equipment failures. Latencies faster than $300 \mathrm{msec}$ and slower than $10,000 \mathrm{msec}$ were also excluded from the analysis $(n=1)$. Analyses were based on the remaining 15,382 trials.

Latencies. Median latencies were calculated for each participant in each condition (aggregated across sessions) and analyzed in a 2 (operation: addition, multiplication) $\times 4$ (format: digit-digit, word-word, digitword, word-digit) $\times 2$ (tie status: nontie, tie) $\times 2$ (problem size: small, large) repeated measures analysis of variance (ANOVA). The ANOVA results are shown in Table 1. Ninety-five percent confidence intervals based on mean-square error values for the appropriate interactions were calculated according to the procedure recommended by Loftus and Masson (1994) and are shown in the figures.

The participants solved small problems more quickly than large problems (1,091 vs. $1,285 \mathrm{msec})$ and tie problems more quickly than nonties (1,115 vs. $1,262 \mathrm{msec})$. As predicted, there was a significant tie $\times$ size interaction, such that the problem-size effect was greater for nonties $(304 \mathrm{msec})$ than for ties $(83 \mathrm{msec})$. Thus, the tie effect in addition and multiplication encompasses both a tie advantage and a tie $\times$ size interaction. Latencies also varied with format such that digit-digit problems were solved more quickly $(1,054 \mathrm{msec})$ than problems presented

Table 1

Experiment 1: ANOVA for Analyses of Solution Latency (in Milliseconds)

\begin{tabular}{llrc}
\hline \multicolumn{1}{c}{ Source } & $d f$ & $M S_{\mathrm{e}}$ & $F$ \\
\hline Main Effects & & & \\
$\quad$ Operation & 1,7 & 37,147 & 1.30 \\
Format & 3,21 & 10,919 & $46.89^{* *}$ \\
Tie status (tie) & 1,7 & 218,404 & $6.35^{*}$ \\
Problem size (size) & 1,7 & 47,839 & $50.24 * *$ \\
Interactions & & & \\
Operation $\times$ format & 3,21 & 4,072 & .77 \\
Operation $\times$ tie & 1,7 & 10,781 & .17 \\
Operation $\times$ size & 1,7 & 18,024 & 2.19 \\
Format $\times$ tie & 3,21 & 7,778 & $26.72 * *$ \\
Format $\times$ size & 3,21 & 2,160 & $4.98^{* *}$ \\
Tie $\times$ size & 1,7 & 43,087 & $18.19^{* *}$ \\
Operation $\times$ format $\times$ tie & 3,21 & 2,523 & 1.30 \\
Operation $\times$ format $\times$ size & 3,21 & 2,898 & $4.59 *$ \\
Operation $\times$ tie $\times$ size & 1,7 & 13,337 & 2.56 \\
Format $\times$ tie $\times$ size & 3,21 & 3,357 & .53 \\
Operation $\times$ format $\times$ tie $\times$ size & 3,21 & 2,230 & $5.91 * *$ \\
\hline * $p .05 . \quad * * p<.01$. & & &
\end{tabular}

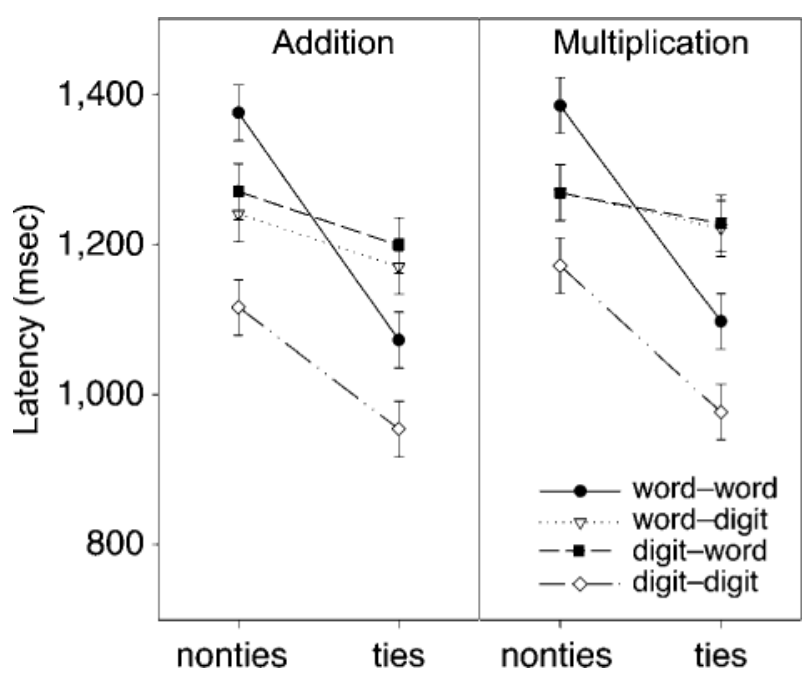

Figure 1. Means of median latencies (in milliseconds) for addition and multiplication problems in Experiment 1: format $\times$ tie status interactions. Whiskers represent $95 \%$ confidence intervals.

in word-word (1,232 msec), digit-word (1,241 msec), and word-digit $(1,225 \mathrm{msec})$ formats $(95 \% C I=77 \mathrm{msec})$.

The main effects of format and tie status were qualified by the significant format $\times$ tie interaction. As shown in Figure 1 for both addition and multiplication, the difference between ties and nonties was greater in the digit-digit and word-word conditions than in the two mixed-format conditions. For multiplication, differences between ties and nonties were not statistically significant in mixed formats. In general, therefore, the tie advantage was substantially reduced in the mixed-format conditions. Blankenberger (2001) interpreted similar patterns of results as evidence that the tie advantage was due entirely to encoding processes.

The encoding-based explanation for the tie effect, however, is qualified by the significant interaction among problem size, tie status, format, and operation, as shown in Figures 2 and 3. Consider the results for addition (Figure 2). The digit-digit condition (left-most panel) shows the typical problem-size $\times$ tie interaction that researchers have noted for 30 years, from Groen and Parkman (1972) to Campbell and Gunter (2002). There was no difference between small ties and nonties and no problem-size effect for ties, whereas the nonties showed a substantial problem-size effect of $240 \mathrm{msec}$, resulting in an overall tie advantage and a tie $\times$ size interaction. Thus, the digit-digit condition serves as a valid baseline for interpretations of the formats that included words.

The central question is: What causes the reduction in the tie advantage in the mixed-format conditions? As shown in the third and fourth panels of Figure 2, latencies on the small ties and small nonties were equal, and the nonties showed problem-size effects very similar to that in the digit-digit condition (234 and $240 \mathrm{msec}$, respectively). However, the ties also showed significant problem-size effects of $87 \mathrm{msec}$ in both mixed-format conditions. These findings indicate that, for addition, the 


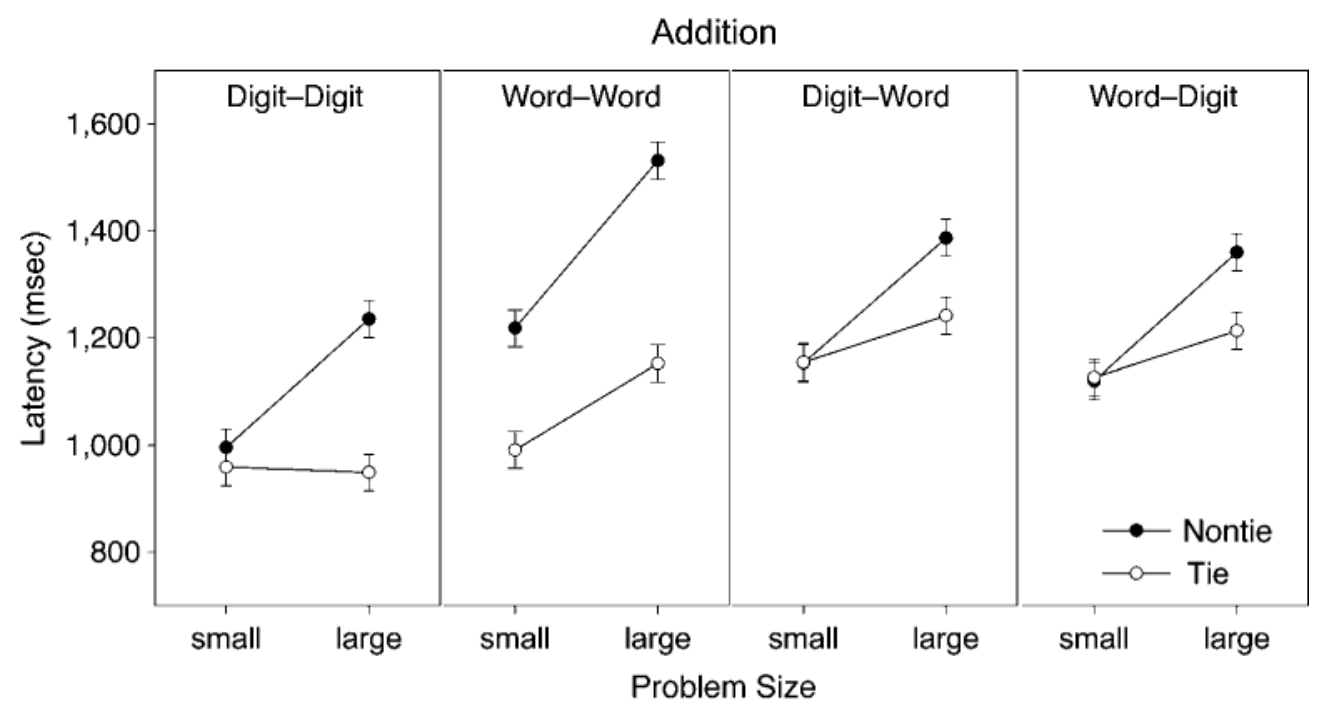

Figure 2. Means of median latencies (in milliseconds) for addition problems in Experiment 1: format $\times$ tie status $\times$ problem-size interactions. Whiskers represent $95 \%$ confidence intervals.

reduction in the tie advantage in mixed-format conditions occurs primarily because the large ties are solved more slowly than expected. Because the small ties were not disadvantaged, however, relative to the nonties, an explanation based on encoding processes is not likely to be correct. Furthermore, the tie advantage was not reversed compared to the results of Campbell and Gunter (2002) for multiplication problems. Thus, an explanation based solely on surface form incompatibilities leading to interference from a comparison process is also not a viable explanation for the results in addition. Instead, the participants may have used procedures other than retrieval to solve large ties more frequently in the mixedformat than in the digit-digit conditions.

In the word-word condition for addition, there was a large advantage for ties. Furthermore, as in the mixedformat condition, the word-word format produced a substantial problem-size effect of $161 \mathrm{msec}$ for ties and an even larger problem-size effect $(313 \mathrm{msec})$ for nonties. Thus, the tie advantage in the word-word condition could be partially due to encoding facilitation, but the pattern of the tie $\times$ problem-size interaction in this condition also provides evidence that tie effects reflect accessbased processes.

In summary, as compared with the digit-digit condition, changing one or both operands to words in addition problems affected problem-size effects for ties substantially, with some effect on nonties when both operands were words. The only condition with clear evidence for an encoding advantage for ties was the word-word condition. The encoding advantage was independent of the tie $\times$ size interaction, however, which was preserved in all three formats that included words. Thus, Blankenberger's (2001) conclusion that the tie advantage is based purely on encoding is not supported by the addition data.
The results for multiplication are shown in Figure 3. Consider the digit-digit condition. The tie $\times$ problemsize interaction consists of both an overall tie advantage (on small and large problems) and a smaller problem-size effect for ties $(152 \mathrm{msec})$ than for nonties $(334 \mathrm{msec})$. What, therefore, causes the reduction in the tie advantage in the mixed conditions? As shown in Figure 3, in the two mixed conditions, small ties were solved more slowly than small nonties, resulting in a reduced problem-size effect of $53 \mathrm{msec}$ in the digit-word condition and $27 \mathrm{msec}$ (not statistically significant) in the word-digit condition. In contrast, although the nonties were solved somewhat more slowly in mixed formats than in the digit-digit format, the problem-size effects were similar to those in the digit-digit condition (356 and $372 \mathrm{msec}$ for digit-word and word-digit, respectively). The participants' performance in the word-word condition was similar to that in the digit-digit condition, although with overall slower latencies, with problem-size effects of $106 \mathrm{msec}$ for ties and $350 \mathrm{msec}$ for nonties. Thus, the reduction in the tie advantage in the mixed formats for multiplication problems was almost entirely due to a relatively greater slowing of small ties compared with all nonties and large ties. These results indicate that a reduction in the tie effect is not primarily due to the absence of an encoding advantage for ties; instead, small ties are actually disadvantaged in the mixed-format conditions as compared with nonties (Campbell \& Gunter, 2002).

Percentage of errors. Because accuracy was emphasized and the participants had plenty of practice, the overall percentage of errors was low (3\%). Nevertheless, for completeness, error data from sessions two through five were analyzed in a 2 (operation: addition, multiplication) $\times 4$ (format: digit-digit, word-word, digitword, word-digit) $\times 2$ (tie status: nontie, tie) $\times 2$ (prob- 


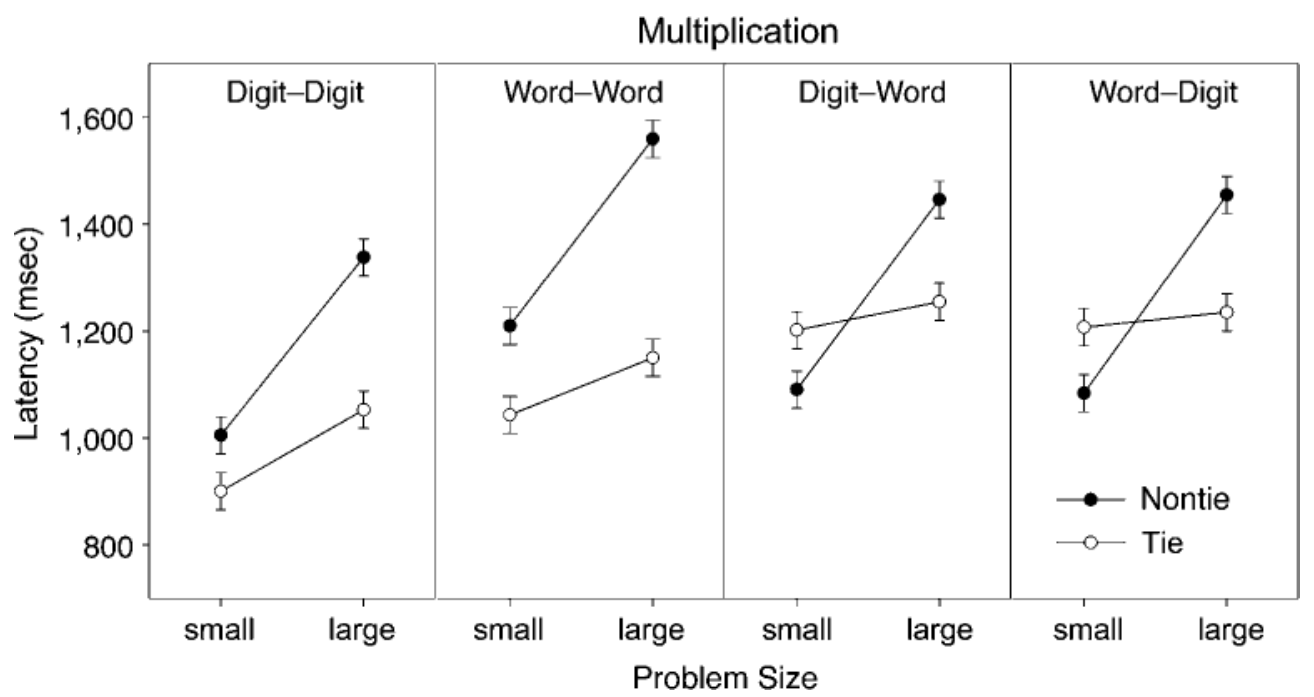

Figure 3. Means of median latencies (in milliseconds) for multiplication problems in Experiment 1: format $\times$ tie status $\times$ problem-size interactions. Whiskers represent $95 \%$ confidence intervals.

lem size: small, large) repeated measures ANOVA. Means are shown in Table 2. Only two effects were significant. First, the problem-size $\times$ tie status interaction was significant $\left[F(1,7)=23.53, M S_{\mathrm{e}}=18.5\right]$. Nontie problems showed a typical problem-size effect in that small nonties were solved with fewer errors than were large nonties ( $1.5 \%$ vs. $4.4 \%)$. In contrast, the participants made more errors on small than on large ties $(3.7 \%$ vs. $1.4 \%)$. Second, as reflected in the format $\times$ tie status interaction $\left[F(3,21)=3.59, M S_{\mathrm{e}}=9.0\right]$, the participants made a similar percentage of errors on nontie problems across the four formats $(3.1,3.0,3.3$, and $2.4 \%$, respectively, for digit-digit, word-word, digit-word, and worddigit) whereas on tie problems, there were fewer errors in the pure than in the mixed conditions $(1.4,1.4,3.6$, and

Table 2

Mean Percentages of Errors in Experiment 1 (Addition and Multiplication) and Experiment 2 (Subtraction and Division)

\begin{tabular}{|c|c|c|c|c|c|c|c|c|}
\hline \multirow[b]{3}{*}{ Size } & \multicolumn{8}{|c|}{ Format } \\
\hline & \multicolumn{2}{|c|}{ Digit-Digit } & \multicolumn{2}{|c|}{ Word-Word } & \multicolumn{2}{|c|}{ Digit-Word } & \multicolumn{2}{|c|}{ Word-Digit } \\
\hline & Nontie & Tie & Nontie & Tie & Nontie & Tie & Nontie & Tie \\
\hline \multicolumn{9}{|c|}{ Addition } \\
\hline Small & 1.0 & 1.7 & 1.7 & 2.4 & 1.5 & 6.4 & 1.4 & 4.0 \\
\hline Large & 3.1 & 0.8 & 3.4 & 1.6 & 3.7 & 0.0 & 3.1 & 0.8 \\
\hline \multicolumn{9}{|c|}{ Multiplication } \\
\hline Small & 1.7 & 2.6 & 1.7 & 0.8 & 1.6 & 4.3 & 1.0 & 7.4 \\
\hline Large & 6.5 & 0.8 & 5.1 & 0.8 & 6.2 & 4.0 & 4.3 & 2.4 \\
\hline \multicolumn{9}{|c|}{ Subtraction } \\
\hline Small & 0.7 & 1.0 & 1.6 & 0.9 & 1.7 & 1.0 & 1.8 & 0.0 \\
\hline Large & 1.9 & 1.0 & 3.6 & 3.0 & 2.1 & 6.3 & 2.7 & 5.8 \\
\hline \multicolumn{9}{|c|}{ Division } \\
\hline Small & 1.8 & 0.0 & 3.4 & 0.0 & 2.8 & 0.0 & 2.2 & 2.7 \\
\hline Large & 3.1 & 1.8 & 4.9 & 1.9 & 3.6 & 1.8 & 3.5 & 0.0 \\
\hline
\end{tabular}

$3.6 \%$, respectively). This finding supports the view that ties are disadvantaged, relative to nonties, in the mixedformat conditions.

\section{Discussion}

The results of Experiment 1 replicated the findings presented by Blankenberger (2001) in that we found a reduction in the tie advantage in mixed-format conditions when the data were averaged across problem size. However, consideration of problem-size effects across the format and tie conditions suggests that this reduction of the tie advantage occurs for reasons other than an encoding advantage in pure formats. The tie $\times$ size interaction (i.e., the finding that the problem-size effect is much larger on nontie than on tie problems) is preserved across all the combinations of format and operation (Figures 2 and 3 ). Thus, the central aspect of the "tie effect," that ties appear to be solved differently than nonties (Groen \& Parkman, 1972), is not eliminated by the format manipulations.

Format did have specific effects on patterns of latencies and errors that suggested the mixed-format condition disrupted performance on ties relative to nonties. Because these effects were most pronounced for multiplication (i.e., the reversed tie effect on small multiplication problems) and were not uniform, they do not support the view that the mixed formats disrupt some sort of general number comparison process that is applied across all problems (Campbell \& Gunter, 2002). For addition, the mixed-format presentation slowed down the processing of all problems (i.e., ties and nonties), suggesting that the presence of word stimuli interfered with performance. The optimal format for addition appears to be the digit-digit condition, suggesting that participants may access addition facts through a direct visual mapping from the digit operands to the answer (Blankenberger 
\& Vorberg, 1997). This hypothesis is supported by the pattern for addition problems in the word-word condition, in that the large nonties are most severely disadvantaged by the word-word format (resulting in a larger problem-size effect for nonties than in the other formats). Campbell and Fugelsang (2001) reported that participants were more likely to use procedures other than memory retrieval on addition problems in word-word format than those in digit-digit format. Thus, the inclusion of word stimuli in the addition problems may have disrupted retrieval of arithmetic facts via a visual code.

For multiplication, the disruption caused by the mixed format was particularly acute for small tie problems. In the two mixed formats, small ties were solved significantly more slowly than small nonties (see also Campbell \& Gunter, 2002), a unique and startling finding relative to all of the other conditions in this article and all published data on ties and problem size. Campbell and Gunter proposed an explanation for this effect based on a disruption to a number comparison process that is circumvented for ties because they have identical operands. This explanation, however, predicts that both addition problems and large ties (in both operations) would show similar or at least proportional disruption. The specificity of the interference to small multiplication ties suggests that some other aspect of processing is being disrupted by the mixed format. This issue will be explored further in the General Discussion section.

In summary, the effect of format on processing of simple addition and multiplication problems is more complex than proposed by Blankenberger (2001). Evidence that the tie advantage is (at least partially) due to encoding facilitation occurred mainly in the word-word format. There was no evidence for encoding facilitation in the digit-digit condition for addition problems, especially because small ties and small nonties were solved equally quickly. The reduction in the tie advantage that Blankenberger reported for mixed format conditions was found, in the present data, to be due largely to changes in the tie $\times$ size interaction as a function of formats. As shown in Figures 2 and 3, the tie $\times$ size interaction changes form but never disappears. Both small and large ties were slower when one or both of the operands were presented as words, but the disruption was greater for large ties in addition and for small ties in multiplication.

\section{EXPERIMENT 2}

Experiment 2 was designed to extend the research on ties in addition and multiplication to subtraction and division. Any tie advantage in these operations cannot be due to an encoding advantage, and therefore patterns of results for these operations across format should provide additional evidence about the source of tie effects. In accord with Campbell and Gunter (2002), ties in subtraction and division were defined as the complements of the tie problems in addition and multiplication. Hence, tie problems were those subtraction and division problems in which the second operand was the same as the answer (e.g., $16 \div 4 ; 10-5$ ). If the tie advantage occurs only as an encoding-based effect, none would be expected for subtraction and division. Campbell and Gunter found tie advantages and the ubiquitous tie $\times$ size interaction in subtraction and division, however. For these operations, the tie $\times$ size interactions were related to the frequent use of calculation procedures on large nonties as compared with ties. On the assumption that word- word or mixed formats should exacerbate differences in selection of procedures (Campbell \& Fugelsang, 2001), we predicted larger tie effects on formats that included words as compared with the digit-digit condition. Note, however, that subtraction and division ties enjoy a potential advantage that relates to the facilitation of answers, rather than to encoding. Consider a problem such as $16 \div$ FOUR. The second operand is identical to, and therefore may facilitate, the answer FOUR. Campbell (1987) showed that nontie multiplication problems such as $6 \times 4=24$, in which an operand is incorporated in the answer, were solved more quickly than equivalent nontie problems that did not have such answer-based advantages, such as $8 \times 3=24$. Thus, we hypothesized that subtraction and division ties would always be faster than nonties because of answer facilitation. Furthermore, because answers to these subtraction and division problems are always a single digit (e.g., four), and because the second operand in these problems is always a single digit (e.g., six), we predicted that most answer facilitation would occur in the conditions in which the second operand was a word (i.e., the word-word and digit-word conditions).

\section{Method}

\section{Participants}

Eight participants were recruited for this experiment; however, one did not complete all of the sessions and so his data were omitted from the analyses. As in Experiment 1, the participants were paid $\$ 90$. The sample included 4 males and 3 females, ranging in age from 20 to 27 years. All were undergraduate students. Six had completed all of their education in Canada, and one had been educated in Kenya and Canada.

\section{Materials}

Arithmetic. The participants solved all 64 subtraction and 64 division problems with subtrahends/divisors and differences/quotients 2 through 9 (i.e., $4-2$ to $18-9$ and $4 \div 2$ to $81 \div 9$ ) in four different formats in each of five sessions. The practice effect for nonties reported by Campbell and Gunter (2002) is not an issue, because reversing the order of the operands does not produce equivalent problems (e.g., $6-4$ vs. $4-6$ ) and complement problems such as $24 \div 6$ vs. $24 \div 4$ do not facilitate one another (Rickard \& Bourne, 1996; Rickard et al., 1994). As in Experiment 1, operands were printed either as Arabic digits or as words in English so that all problems were presented in two pure formats (e.g., $4 \div 2$, FOUR $\div$ TWO) and two mixed formats (e.g., $4 \div$ TWO, FOUR $\div 2$ ). The participants, therefore, solved 512 simple arithmetic problems $(128 \times$ 4 formats), divided into 8 blocks of 64 , in each session. Format (digit-digit, word-word, digit-word, word-digit) and operation (subtraction, division) varied randomly from trial to trial.

Naming. To test production (naming) latencies, the eight possible correct answers to the subtraction and division problems were 
presented in random order in digit format. The participants named each digit three times, for a total of 24 naming trials per session.

Fluency test and background questionnaire. The participants completed the addition and subtraction-multiplication subtests of the French Kit (French, Ekstrom, \& Price, 1963) and the background questionnaire, as in Experiment 1.

\section{Procedure}

The apparatus and procedure were identical to those in Experiment 1.

\section{Results}

\section{Questionnaire and Arithmetic Fluency}

The participants described a variety of procedures for solving simple subtraction and division problems, including recasting division as multiplication (e.g., $8 \times{ }_{-}=$ $72)$ and subtraction as addition $\left(9+_{-}=16\right)$, decomposition (e.g., $17-9=[17-10]+1)$, counting backward, and a nines rule (one for subtraction and one for division). These participants reported using retrieval to solve simple arithmetic problems more frequently than did the participants in Experiment 1. Most of the participants reported that they used procedures other than retrieval never or rarely for subtraction ( 5 out of 7 ), division ( 6 out of 7 ), addition ( 5 out of 7 ), and multiplication (7 out of 7). As a group, they were highly skilled at multidigit arithmetic. The total scores on the arithmetic fluency test ranged from 69 to 111, with a mean of 95 ( $S D=$ 17.4). Thus, as a group, the participants in this experiment had higher arithmetic fluency than the participants in Experiment 1.

\section{Naming}

The participants completed a total of 840 naming trials. In contrast to Experiment 1, the answers do not differ across tie status; each answer from 2 through 9 occurs both as a tie and as a nontie. For problem size, the answer "two" occurs only in small problems, but all other answers occur in both small and large problem sets. Thus, patterns of naming latencies would not affect arithmetic performance and were not considered further.

\section{Arithmetic}

The participants solved a total of 17,920 arithmetic trials. Session one was considered practice and excluded from the analysis. For the remaining four sessions, 342 trials were errors, and 420 trials were invalid, due either to inadvertent verbalizations by the participant or to equipment failures. Furthermore, latencies faster than $300 \mathrm{msec}$ and slower than $10,000 \mathrm{msec}$ were excluded from the analyses $(n=109)$. For the remaining trials $(n=13,465)$, median latencies were calculated for each participant in each condition (aggregating across sessions) and analyzed in a 2 (operation: subtraction, division) $\times 4$ (format: digit-digit, word-word, digit-word, word-digit) $\times 2$ (tie status: nontie, tie) $\times 2$ (problem size: small, large) repeated measures ANOVA. The ANOVA results are shown in Table 2. Ninety-five percent confidence intervals based on mean-square error
Table 3

Experiment 2: ANOVA for Analyses of Solution Latency (in Milliseconds)

\begin{tabular}{|c|c|c|c|}
\hline Source & $d f$ & $M S_{\mathrm{e}}$ & $F$ \\
\hline \multicolumn{4}{|l|}{ Main Effects } \\
\hline Operation & 1,6 & 27,403 & .37 \\
\hline Format & 3,18 & 17,266 & $38.15^{* *}$ \\
\hline Tie status (tie) & 1,6 & 87,833 & $15.34 * *$ \\
\hline Problem size (size) & 1,6 & 77,484 & $19.97 * *$ \\
\hline \multicolumn{4}{|l|}{ Interactions } \\
\hline Operation $\times$ format & 3,18 & 2,492 & $13.90 * *$ \\
\hline Operation $\times$ tie & 1,6 & 2,908 & $7.65^{*}$ \\
\hline Operation $\times$ size & 1,6 & 18,898 & $8.90 *$ \\
\hline Format $\times$ tie & 3,18 & 5,555 & $5.94 * *$ \\
\hline Format $\times$ size & 3,18 & 5,284 & $15.00 * *$ \\
\hline Tie $\times$ size & 1,6 & 42,403 & $6.36^{*}$ \\
\hline Operation $\times$ format $\times$ tie & 3,18 & 3,984 & 1.92 \\
\hline Operation $\times$ format $\times$ size & 3,18 & 2,962 & 1.56 \\
\hline Operation $\times$ tie $\times$ size & 1,6 & 12,237 & .01 \\
\hline Format $\times$ tie $\times$ size & 3,18 & 3,472 & 1.49 \\
\hline Operation $\times$ format $\times$ tie $\times$ size & 3,18 & 3,748 & $4.26^{*}$ \\
\hline
\end{tabular}

values for the appropriate interactions were calculated according to the procedure recommended by Loftus and Masson (1994) and are shown in the figures.

The participants solved small problems more quickly than large problems (926 vs. 1,093 msec, respectively), replicating the problem-size effect identified in the literature. They also solved tie problems more quickly than nonties ( 932 vs. $1,087 \mathrm{msec}$, respectively), indicating that the tie advantage was robust for these operations. Furthermore, problem size interacted with tie status such that participants showed larger problem-size effects on nontie than on tie problems (236 vs. $97 \mathrm{msec}$, respectively). Thus, subtraction and division show patterns of tie advantage and tie $\times$ size interactions that are similar to those found for addition and multiplication.

Latencies varied with formats. The participants solved word-word problems $(1,139 \mathrm{msec})$ significantly more slowly than digit-digit $(880 \mathrm{msec})$ or digit-word problems (979 msec; $95 \% C I=104 \mathrm{msec})$. They also solved digit-digit problems more quickly than word-digit problems $(1,040 \mathrm{msec})$, whereas digit-word problems were not significantly different from either of those conditions. The effect of format varied across operation, however. As shown in Figure 4, format costs (i.e., differences between the digit-digit format and each of the other conditions) were generally smaller for subtraction than for division. Furthermore, the two mixed formats were solved equally quickly in subtraction, and the additional cost of the word-word format was small. For division, the two formats with an initial word showed larger costs than the digit-word format. All of the words in these subtraction problems were probably relatively familiar in written form (i.e., the largest was EIGHTEEN) and consisted of single words (e.g., EIGHT, TWELVE, EIGHTEEN), whereas the words in division problems were less familiar in their written form and consisted of compound words as com- 


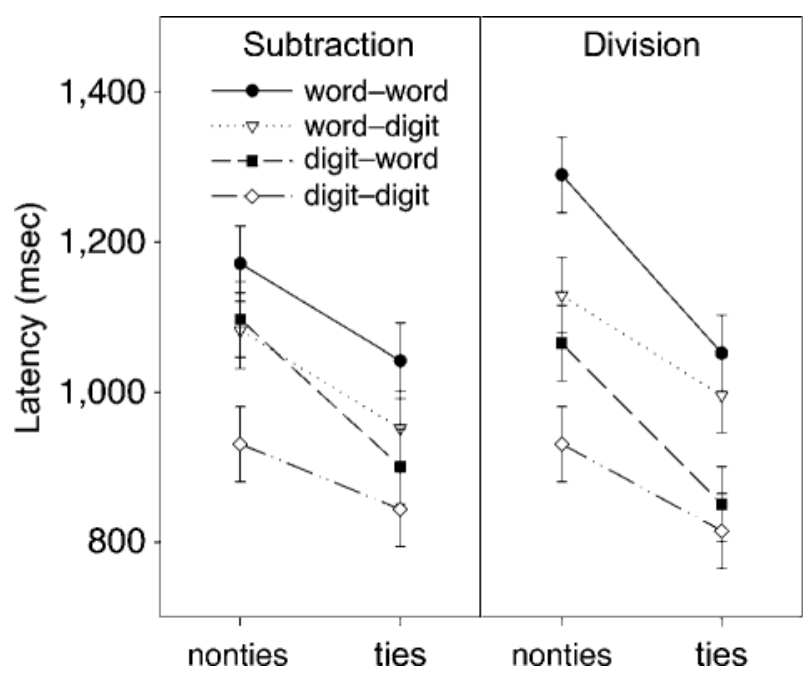

Figure 4. Means of median latencies (in milliseconds) for subtraction and division problems in Experiment 2: format $\times$ tie status interactions. Whiskers represent $95 \%$ confidence intervals.

pared with those in division problems (e.g., TWENTYSEVEN, SIXTY-FOUR, EIGHTY-ONE). More generally, these results suggest that costs of word formats in arithmetic may be related to the familiarity of the stimuli as words or to word length.

As in Experiment 1, there was a format $\times$ tie interaction, but the form of the interaction was quite different. Ties were faster than nonties in all formats but, as shown in Figure 4, word-word and digit-word formats showed greater tie advantages than did digit-digit and word-digit formats. This pattern is consistent with the hypothesis that a portion of the tie advantage in subtraction and division occurs because the presentation of the second operand as a word (e.g., $4-$ TWO or FOUR $\div$ TWO) facilitates the verbal response (i.e., TWO) more than does the presentation of the second operand as a digit (e.g., $4-2$ or FOUR $\div 2$ ).

All significant two-way interactions were qualified by the significant four-way interaction, as shown in Figures 5 and 6. As in Experiment 1, the tie $\times$ size interaction was preserved in all combinations of operation and format. Thus, the presence of the tie $\times$ size interaction was not dependent upon format, although there are differences in the form of the interaction across conditions.

Consider participants' performance on subtraction problems (Figure 5). For digit-digit problems, there was no significant difference between small ties and small nonties, and the problem-size effect was larger for nonties $(255 \mathrm{msec})$ than for ties $(145 \mathrm{msec})$. The pattern was very similar in the word-digit condition (e.g., FOURTEEN - 7) in that nonties showed a problem-size effect of $279 \mathrm{msec}$ as compared with the $152 \mathrm{msec}$ effect shown for ties. In the digit-word condition, although the problemsize effect on nonties $(258 \mathrm{msec})$ was very similar to the digit-digit and word-digit conditions, ties showed a smaller problem-size effect $(44 \mathrm{msec})$ that was not sta- tistically significant. This pattern suggests that in the digit-word condition (e.g., 14 - seven), facilitation of the answer from the word was greater than when the second operand was presented as a digit.

In the word-word condition, ties were significantly faster than nonties. Furthermore, although the tie $\times$ size interaction was maintained, both problem categories showed large effects of problem size: $339 \mathrm{msec}$ for nonties and $268 \mathrm{msec}$ for ties. Thus, the word-word format may have disrupted the access-based advantage enjoyed by ties, an effect that was moderated by the presence of an advantage for ties in which the second operand facilitated the answer.

As shown in Figure 6 for division problems, tie effects were preserved across formats. In the digit-digit condition, there was a significant difference between small ties and small nonties, no problem-size effect for ties (6 msec), and a significant (101 msec) effect for nonties. In the digit-word condition, the pattern was very similar, except that ties showed a larger overall advantage. The problem-size effect for ties was not significant $(9 \mathrm{msec})$ and for nonties, it was similar to that in the digit-digit condition $(121 \mathrm{msec})$. Comparing across the digit-digit and digit-word conditions indicates that tie latencies were not significantly different, suggesting that the presentation of the second operand as a word did not disrupt processing of these tie problems. This is in contrast to Experiment 1, in which the presence of a word resulted in slower latencies for ties as well as for nonties.

In the word-digit condition (e.g., SEVENTY-TWO $\div 8$ ), the participants showed significant problem-size effects of $105 \mathrm{msec}$ for ties and $198 \mathrm{msec}$ for nonties. In contrast, although latencies in the word-word condition were slower than in the other conditions, ties did not show a significant problem-size effect (43 msec) as compared with nonties $(300 \mathrm{msec})$. The answer facilitation resulting from presenting the second operand as a word for ties (e.g., SIXTEEN $\div$ FOUR $=$ FOUR) appears to have reduced the access-based costs associated with the word formats.

In summary, nontie latencies were related to the presence of words (more words, slower latencies), with the effect being greater when the first operand was a word than when the second operand was a word. The substantially larger problem-size effect for nonties in the word-word condition as compared with nonties in the other conditions, however, suggests that solving those problems presented further difficulties for the participants.

Percentage of errors. The overall percentage of errors was low (mean of $2.1 \%$ ); nevertheless, analyses are presented for the sake of completeness. As for latencies, error percentages were analyzed in a 2 (operation: subtraction, division) $\times 4$ (format: digit-digit, word-word, digit-word, word-digit) $\times 2$ (tie status: nontie, tie) $\times 2$ (problem size: small, large) repeated measures ANOVA. Means are shown in Table 2. Participants made fewer errors on small than on large problems $(1.3 \%$ vs. $2.9 \%)$ $\left[F(1,6)=14.70, M S_{\mathrm{e}}=9.51, p<.01\right]$. They also made 


\section{Subtraction}

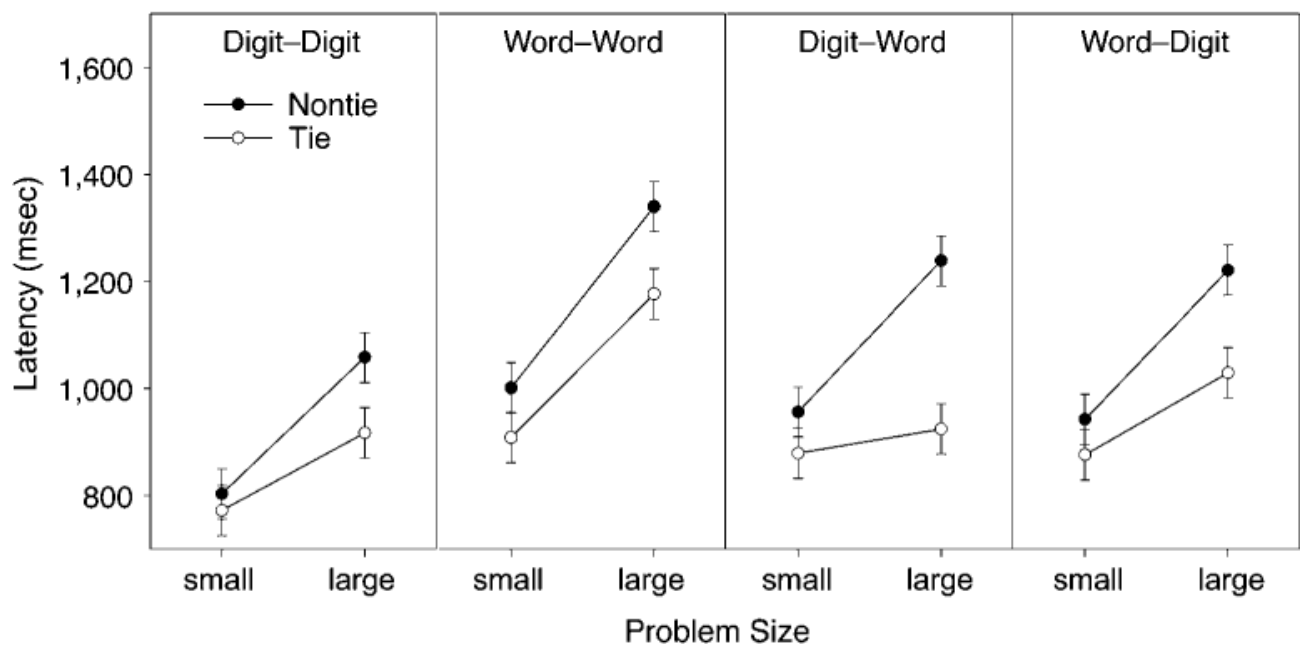

Figure 5. Means of median latencies (in milliseconds) for subtraction problems in Experiment 2: format $\times$ tie status $\times$ problem-size interactions. Whiskers represent $95 \%$ confidence intervals.

somewhat fewer errors on nonties than on ties $(1.7 \%$ vs. $2.6 \%)\left[F(1,6)=5.82, M S_{\mathrm{e}}=7.61, p=.052\right]$. The only other significant effect was the tie $\times$ size interaction $\left[F(1,6)=6.92, M S_{\mathrm{e}}=1.44, p<.05\right]$. The problem-size effect was smaller for nonties $(1.2 \%)$ than for ties $(2.0 \%)$. These patterns do not compromise the latency analyses and will not be discussed further.

\section{Discussion}

Experiment 2 was designed to further explore tie advantages and tie $\times$ size interactions for subtraction and division. Few reports of tie effects have been published for these operations (Campbell, 1997; Campbell \& Gunter, 2002), as compared with addition and multiplication. Tie advantages were found for both subtraction and division, with the patterns of tie $\times$ size interactions in digit-digit conditions very similar to those for addition and multiplication. Tie effects in subtraction and division cannot be due to encoding facilitation but there was evidence for answer facilitation, in that tie problems with the second operand presented as a word (e.g., $81 \div$ NINE) showed an even larger tie advantage than problems in which the second operand was a digit. This advantage

Division

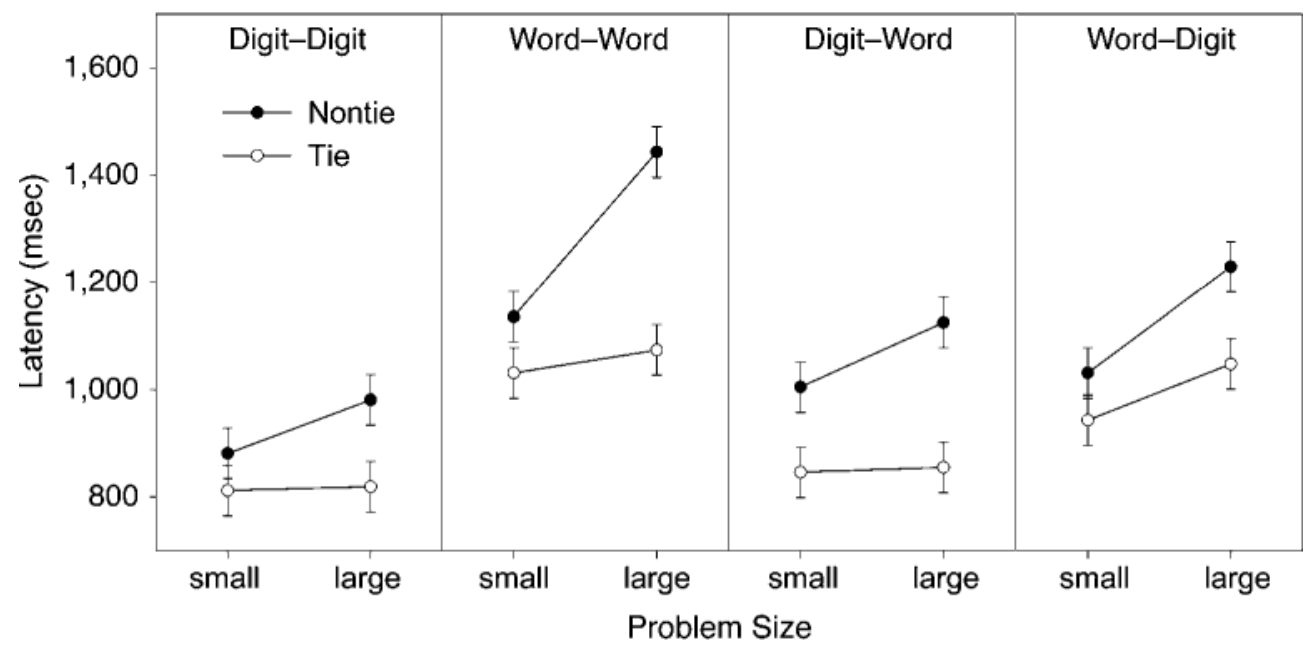

Figure 6. Means of median latencies (in milliseconds) for division problems in Experiment 2: format $\times$ tie status $\times$ problem-size interactions. Whiskers represent $95 \%$ confidence intervals. 
would presumably be confined mainly to tasks in which the answers are spoken aloud. Furthermore, presentation of the operands in word form moderated the tie $\times$ size interactions in both subtraction and division, indicating that access-based processing was influenced by changes in format.

\section{GENERAL DISCUSSION}

Our goal was to explore the tie effect in simple arithmetic. Researchers have consistently found that, in addition and multiplication, problems with repeated operands such as $3+3$ or $4 \times 4$ are solved more quickly than comparable nonties such as $3+4$ or $4 \times 3$. Furthermore, tie status interacts with problem size, such that the problemsize effect is either nonexistent (addition) or substantially smaller (multiplication) for ties as compared with nonties. Blankenberger (2001) proposed that the tie advantage in addition and multiplication occurs because participants can encode two identical physical stimuli more quickly than two different stimuli. In the present research, we found minimal evidence that the overall tie advantage is related to encoding. Instead, we found that the tie $\times$ size interaction, a pattern that is assumed to represent differential calculation and memory processes for ties and nonties, was preserved across format conditions. Thus, we conclude that the tie advantage is not primarily an encoding effect.

We did not find support for the encoding hypothesis in addition and multiplication when the digit-digit condition (the standard format) was compared with mixed formats. Nevertheless, the overall tie advantage for these operations was greater in the word-word format condition than in the others. Because latencies also tended to be slower in the word-word conditions, it seems likely that the repetition of the word stimulus in ties (e.g., FIVE + FIVE Vs. FIVE + SIX) provided an encoding advantage. Presumably, this encoding advantage was greater in the word-word condition because people are so much slower to process words in arithmetic problems than digits (see Campbell, 1994). For division and subtraction, tie advantages were greater when the second operand was a word than when it was a digit (i.e., the word-word and digit-word conditions). Here, the facilitation was presumably due to increased activation of the answer code because the required response was always a spoken word. Campbell (1987) also described answer facilitation for problems such as $6 \times 4=$ TWENTY-FOUR, although he did not test word-word or mixed-format conditions. Despite these effects of facilitation in wordword and digit-word conditions, however, the tie $\times$ size interactions were preserved (or augmented), indicating that any facilitation due to encoding did not affect the underlying sources for differential processing of ties versus nonties. Thus, these data support the view that tie effects, particularly those revealed in tie $\times$ size interactions, are related to calculation and memory processes involved in accessing the answers to the problems.
Various explanations have been proposed for the observed interaction between tie status and problem size, including differential familiarity, interference, and selection of procedures across the two problem categories. On the assumption that using word-word or mixed formats is likely to influence participants' solution processes (Campbell \& Fugelsang, 2001), the present results provide support for the explanation that the tie $\times$ size interaction is, at least in part, based on differential selection of procedures. Campbell and Fugelsang found that when participants solved problems presented in word-word formats, they reported using fewer direct retrieval solutions than when they solved the same problems presented in digit-digit formats. Because retrieval is faster than alternative solution procedures (such as counting or reformulating the problem), and because alternative solution procedures are even slower with large than with small problems, one consequence of using retrieval less frequently is an increased problem-size effect (Campbell \& Fugelsang, 2001). Any such changes from retrieval to other solutions would affect ties more than nonties, because participants typically do not use procedures other than retrieval when ties are presented in the preferred digit-digit format (LeFevre, Bisanz, et al., 1996). Thus, the possibility that participants use retrieval less frequently in mixed-format conditions than in the digit-digit condition would predict the observed pattern of results, that is, larger problem-size effects and more disruption on ties than on nonties.

More generally, we might expect that the most disruption from unusual formats in a given operation will occur for those problems that are most often solved via direct retrieval when problems are presented in the preferred and typical digit-digit format. In the present research (see also Campbell \& Gunter, 2002), the most disrupted category of problems was small ties in multiplication. The tie effect was not simply eliminated in mixed conditions for these problems, but actually reversed. This finding suggests that small multiplication ties $(2 \times 2$, $3 \times 3,4 \times 4$, and $5 \times 5$ ) enjoy a unique status among multiplication problems (LeFevre, Bisanz, et al., 1996). In contrast, small ties and nonties were equally disrupted in addition, suggesting that all small addition problems are solved most efficiently in digit-digit format. Further, large addition ties were disrupted more than large nonties, in support of the view that people use direct retrieval to solve large addition ties, but a greater mix of procedures on large nonties. These results are consistent with Blankenberger and Vorberg's (1997) conclusion that addition problems enjoy a privileged solution route in digit-digit format.

In summary, the present results showed that the tie effect that has been observed for over 30 years in the study of simple arithmetic (Groen \& Parkman, 1972) is not solely or even largely due to an encoding advantage for repeated operands. The preservation of the tie $\times$ size interaction across format conditions indicates that a more complex explanation for patterns of tie and tie $X$ 
size effects is required. There was evidence for an encoding advantage when problems were presented as wordword problems, but in all combinations of format and operation, the tie effect was best understood as a tie $\times$ size interaction such that ties show less of a problem-size effect than do nonties. Because this interaction is assumed to reflect differences in how ties and nonties are solved, and because the interaction was preserved or augmented across mixed format conditions, we conclude that the tie effect is related to calculation and memory access and not to encoding.

\section{REFERENCES}

Ashcraft, M. H. (1992). Cognitive arithmetic: A review of data and theory. Cognition, 44, 75-106.

AshCRAFT, M. H. (1995). Cognitive psychology and simple arithmetic: A review and summary of new directions. Mathematical Cognition, 1, 3-34.

AshCraft, M. H., \& Christy, K. S. (1995). The frequency of arithmetic facts in elementary texts: Addition and multiplication in grades 1-6. Journal for Research in Mathematics Education, 26, 396-421.

BLANKENBERGER, S. (2001). The arithmetic tie effect is mainly encodingbased. Cognition, 82, B15-B24.

Blankenberger, S., \& Vorberg, D. (1997). The single-format assumption in arithmetic fact retrieval. Journal of Experimental Psychology: Learning, Memory, \& Cognition, 23, 721-738.

Butterworth, B., Zorzi, M., Girelli, L., \& JoncKheere, A. R. (2001). Storage and retrieval of addition facts: The role of number comparison. Quarterly Journal of Experimental Psychology, 54A, 1005-1029.

CAMPBELL, J. I. D. (1987). Network interference and mental multiplication. Journal of Experimental Psychology: Learning, Memory, \& Cognition, 13, 109-123.

CAMPBELL, J. I. D. (1994). Architectures for numerical cognition. Cognition, 53, 1-44.

CAMPBELL, J. I. D. (1995). Mechanisms of simple addition and multiplication: A modified network-interference theory and simulation. Mathematical Cognition, 1, 121-164.

CAMPBELL, J. I. D. (1997). On the relation between skilled performance of simple division and multiplication. Journal of Experimental Psychology: Learning, Memory, \& Cognition, 23, 1140-1159.

CAMPBELL, J. I. D. (1998). Linguistic influences in cognitive arithmetic: Comment on Noël, Fias, and Brysbaert (1997). Cognition, 67, 353-364.

CAmpbell, J. I .D. (1999). Division by multiplication. Memory \& Cognition, 27, 791-802.

CAMPBel, J. I. D., \& Fugelsang, J. (2001). Strategy choice for arithmetic verification: Effects of numerical surface form. Cognition, 80, B21-B30.

CAmpbell, J. I. D., \& Gunter, R. (2002). Calculation, culture, and the repeated operand effect. Cognition, 86, 71-96.

Campbell, J. I. D., \& Xue, Q. (2001). Cognitive arithmetic across cultures. Journal of Experimental Psychology: General, 130, 299-315.

French, J. W., Ekstrom, R. B., \& Price, I. A. (1963). Kit of reference tests for cognitive factors. Princeton, NJ: Educational Testing Service.
GeARY, D. C. (1996). The problem-size effect in mental addition: Developmental and cross-national trends. Mathematical Cognition, 2, 63-93.

Groen, G. J., \& Parkman, J. M. (1972). A chronometric analysis of simple arithmetic. Psychological Review, 79, 329-343.

HamanN, M. S., \& AshCRAFT, M. H. (1986). Textbook presentations of basic arithmetic facts. Cognition \& Instruction, 3, 173-192.

Неснт, S. A. (1999). Individual solution processes while solving addition and multiplication math facts in adults. Memory \& Cognition, 27, 1097-1107.

LeFevre, J., Bisanz, J., Daley, K. E., Buffone, L., Greenham, S. L., \& SADESKY, G. S. (1996). Multiple routes to solution of single-digit multiplication problems. Journal of Experimental Psychology: General, 125, 284-306.

LeFevrE, J., \& LiU, J. (1997). Numerical cognition: Single-digit multiplication skills of adults from China and Canada. Mathematical Cognition, 3, 31-62.

LeFEVRe, J., SADESKY, G. S., \& BisanZ, J. (1996). Selection of procedures in mental addition: Reassessing the problem-size effect in adults. Journal of Experimental Psychology: Learning, Memory, \& Cognition, 22, 216-230.

LeFevre, J., SMith-Chant, B. L., Hiscock, K., Daley, K. E., \& MorRIS, J. (2003). Young adults' strategic choices in simple arithmetic: Implications for the development of mathematical representations. In A. J. Baroody \& A. Dowker (Eds.), The development of arithmetic concepts and skills: Constructing adaptive expertise (pp. 203-228). Mahwah, NJ: Erlbaum.

Loftus, G. R., \& MAsson, M. E. J. (1994). Using confidence intervals in within-subject designs. Psychonomic Bulletin \& Review, 1, 476490.

Miller, K., Perlmutter, M., \& Keating, D. (1984). Cognitive arithmetic: Comparison of operations. Journal of Experimental Psychology: Learning, Memory, \& Cognition, 10, 46-60.

NoËL, M., Fias, W., \& BrysbaerT, M. (1997). About the influence of presentation format on arithmetical fact-retrieval processes. Cognition, 63, 335-374.

Nö̈L, M., RoberT, A., \& Brysbaert, M. (1998). Does language really matter when doing arithmetic? Reply to Campbell (1998). Cognition, 67, 365-373.

PenNer-Wilger, M., Leth-Steensen, C., \& LeFevre, J. (2002). Decomposing the problem-size effect: A comparison of response time distributions across cultures. Memory \& Cognition, 30, 1160-1167.

Rickard, T. C., \& Bourne, JR., L. E. (1996). Some tests of an identical elements model of basic arithmetic skills. Journal of Experimental Psychology: Learning, Memory, \& Cognition, 22, 1281-1295.

RicKard, T. C., Healy, A. F., \& Bourne, JR., L. E. (1994). On the representation of arithmetic facts: Operand order, symbol, and operation transfer effects. Journal of Experimental Psychology: Learning, Memory, \& Cognition, 20, 1139-1153.

SIEGLER, R. S., \& SHRAGER, J. (1984). Strategy choices in addition and subtraction: How do children know what to do? In C. Sophian (Ed.), The origins of cognitive skills. Hillsdale, NJ: Erlbaum.

Smith-Chant, B. L., \& LeFevre, J. (2003). Doing as they are told and telling it like it is : Self-reports in mental arithmetic. Memory \& Cognition, 31, 516-528.

ZBRODOFF, N. J. (1995). Why is $9+7$ harder than $2+3$ ? Strength and interference as explanations of the problem-size effect. Memory \& Cognition, 23, 689-700. 